\title{
Personal protection and delivery of rhinologic and endoscopic skull base procedures during the COVID-19 outbreak: ERS endorsed advises*
}

L. Van Gerven', PW. Hellings ${ }^{1,2,3}$, T. Cox ${ }^{4}$, W.J. Fokkens ${ }^{3}$, C. Hopkins ${ }^{5}$, V. Hox , M. Jorissen ${ }^{1}$, A. Schuermans ${ }^{7,8}$, P. Sinonquel ${ }^{9}$, K. Speleman ${ }^{10}$, V. Vander Poorten ${ }^{1,11}$, K. Van Gool' ${ }^{12}$, T. Van Zele'2, I. Alobid ${ }^{13}$
Rhinology 58: 3, 289 - 294, 2020

https://doi.org/10.4193/Rhin20.119

*Received for publication:

March 30, 2020

Accepted: April 6, 2020

2 University of Ghent, Department of Otorhinolaryngology, Laboratory of Upper Airways Research, Ghent, Belgium

${ }^{3}$ Academic Medical Center Amsterdam, Department of Otorhinolaryngology, Amsterdam, the Netherlands

${ }^{4}$ Jessa Hospital, Department of Otorhinolaryngology, Head \& Neck Surgery, Hasselt, Belgium

5 Guy's and St Thomas' Hospitals, Department of Otorhinolaryngology, London, United Kingdom

${ }^{6}$ Cliniques Universitaires Saint-Luc, Department of Otorhinolaryngology, Head \& Neck Surgery, Brussels, Belgium

University Hospitals Leuven, Department of Infection Control and Epidemiology, Leuven, Belgium

${ }^{3}$ KU Leuven, Department of Public Health and Primary Care, Leuven, Belgium

9 University Hospitals Leuven, Department of Gastro-enterology and Hepatology, Leuven, Belgium

${ }^{10}$ Sint-Jan Hospital, Department of Otorhinolaryngology, Head \& Neck Surgery, Bruges, Belgium

1 University Hospitals Leuven, Department of Oncology, section Head and Neck Oncology, Leuven, Belgium

12 University Hospital Antwerp, Department of Otorhinolaryngology, Head \& Neck Surgery, Antwerp, Belgium

${ }^{13}$ Hospital Clinic Barcelona, Rhinology Unit \& Smell Clinic, ENT Department, Centro Médico TEKNON, Barcelona, Spain

\begin{abstract}
On March 11th 2020, the World Health Organization (WHO) declared COVID-19 pandemic, with subsequent profound impact on the entire health care system. During the COVID-19 outbreak, activities in the rhinology outpatient clinic and operation rooms are limited to emergency care only. Health care practitioners are faced with the need to perform rhinological and skull base emergency procedures in patients with a positive or unknown COVID-19 status.

This article aims to provide recommendations and relevant information for rhinologists, based on the limited amount of (anecdotal) data, to guarantee high-quality patient care and adequate levels of infection prevention in the rhinology clinic.
\end{abstract}

Key words: SARS-CoV-2, COVID-19, personal protective equipment, rhinology, skull base surgery, infection prevention, endoscopic surgery

\section{Introduction}

On December 31st, 2019, the WHO was being informed by the authorities of the People's Republic of China about several cases of pneumonia of unknown cause in Wuhan, a city of 11 million people, in the province of Hubei (China) $)^{(1)}$. One week later, China confirmed that the cases were due to a new coronavirus, named SARS-CoV-2 ${ }^{(2)}$. The SARS-CoV-2 virus and the disease it causes, termed "coronavirus disease 2019" (abbreviated COVID-19) spread during the following weeks and reached Europe beginning of February 2020 with northern Italy and more specific the province of Lombardy as new epicenter of the pandemic (97.689 cases according to Johns Hopkins Coronavirus Resource center at the end of March) ${ }^{(3)}$. On March 12th, the Belgian Government issued a decree with immediate effect. The decree involved changes to the organization of health care installations, staff and services ${ }^{(4)}$. In line with the new legal situation, the respective health care departments modified their services, often restricting medical activities to emergency care only. We aim to provide recommendations and precautions, based on the limited amount of data, on the best way to safely perform rhinological examinations and procedures, for both patients and practitioners within the current regulatory context. Transmission of SARS-CoV-2 is primarily through droplet spread $^{(5)}$. Therefore, otorhinolaryngologists/ ENT doctors are 
Table 1. Overview of suggested recommendations for PPE during endoscopic endonasal procedures depending on the COVID-19 status of the patient.

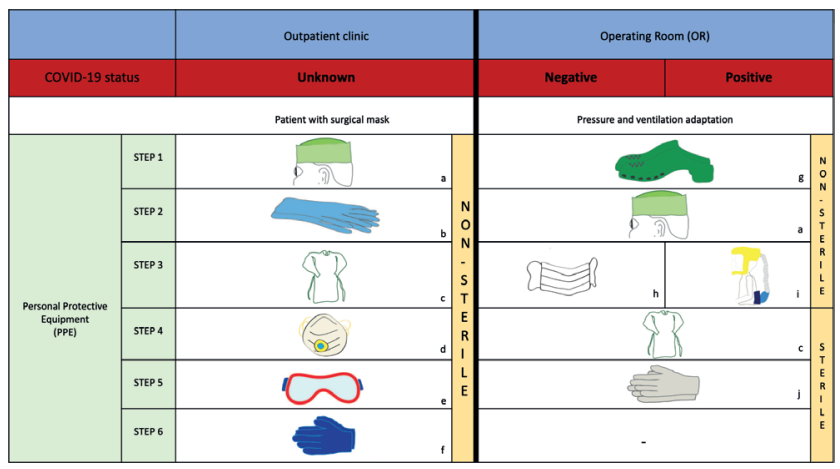

( $a$ = surgical cap, $b=$ long gloves, $c=$ impermeable gown, $d=$ FFP2 mask, $\mathrm{e}=$ goggles, $\mathrm{f}=$ short gloves, $\mathrm{g}=$ scrubs, $\mathrm{h}=$ surgical mask, $\mathrm{i}=$ powered, air-purifying respirator, $\mathrm{j}=$ sterile gloves).

exposed to a high reservoir of viral load as they are dealing with pathology in the upper respiratory tract ${ }^{(6)}$. Overall, 1 in 7 new COVID-19 cases affects a health care worker, with presumably higher rates in intensive care and emergency doctors, anesthesiologists, ENT surgeons and pulmonologists. Recently, Zou et al. showed higher viral loads soon after symptom-onset and higher viral loads in the nose compared to the throat ${ }^{(5)}$. The viral load that was detected in asymptomatic patients was similar to that in symptomatic patients, which suggests high transmission-potential of asymptomatic (or minimally symptomatic) patients ${ }^{(5)}$. Additionally, if viral particles become aerosolized, they stay in the air for at least 3 hours ${ }^{(7)}$. Recent anecdotal data from China, Iran, Italy, Australia and most recently the UK suggest that ENT surgeons and rhinologists in particular, are at increased risk to become infected. Additionally, a report from the Stanford group underlined these findings and provided some recommendations for Otorhinolaryngologists ${ }^{(8)}$. This is most likely due to aerosol formation from drilling, rinsing and aspiration during nasal endoscopy and endoscopic sinus surgery ${ }^{(9)}$. Fatalities among ENT doctors have been reported. Therefore, the need has arisen to increase awareness and to ask to be vigilant in the protection of health care providers and team members with the necessary personal protective equipment (PPE).

\section{Special report}

Procedures at the outpatient rhinology clinic

Based on the above-mentioned observations, diagnostic procedures involving upper airway manipulation like anterior rhinoscopy and even more, nasal endoscopy, should be considered high risk for viral transmission, and therefore limited to patients with an urgent need to be examined during the initial phase of the COVID-19 outbreak ${ }^{(10)}$. Urgent rhinologic cases include patients with recurrent or severe epistaxis, suspicion of complicated acute/chronic rhinosinusitis (intra-orbital or intracranial), sinonasal neoplasms, and/or immediate (CSF-leak) or late (mucocele with loss of vision) postoperative complications. Additionally, post-operative care, such as removal of packing and silastic sheets, has to be performed safely.

Before clinical examination, it is recommended to question all patients about contact with confirmed COVID-19 patients, fever, respiratory symptoms and recent sudden loss of smell and/or taste. Several reports mention sudden anosmia as an early sign of COVID-19 infection, often preceding other symptoms ${ }^{(11-13)}$.

People are most contagious in the $72 \mathrm{~h}$ after the development of symptoms, so early recognition can prevent further spreading ${ }^{(5)}$. The rules for social distancing need to be applied in the waiting room. Visiting patients should be informed, chairs provided at the required distance and the number of patients as well as the waiting time should be limited as much as possible. Preferentially, patients should not be accompanied by relatives or friends. It is advised to try to predict the necessary material and prepare in such way to avoid unnecessary movement with already contaminated equipment once the clinical examination has started. During clinical examination and more specific nasal endoscopy, distance between the ENT-physician and patient can be maximized by using a tower with camera, screen and light source instead of using an eyepiece to avoid (too) close physical contact. Also, manipulations should be limited if possible, i.e. nasal inspection versus debridement with suction and/or forceps. The use of (local anesthetic) spray can be replaced by alternatives such as soaked pledgets because atomized anesthesia can aerosolize the virus ${ }^{(14)}$.

Since the COVID-19 status of patients consulting the outpatient rhinology clinic is often unknown and since the risk of transmission through clinical procedures is high, wearing adequate PPE is mandatory, including cap, (long) nitrile gloves, impermeable gown, FFP2 mask and goggles/ face visor for the person who is performing the clinical examination (World Health Organization. Rational Use of Personal Protective Equipment for Coronavirus Disease 2019 (COVID-19); 2020. https://apps. who.int/iris/bitstream/handle/10665/331215/WHO-2019-nCovIPCPPE_use2020.1-eng.pdf) (Table 1). Scarcity of masks and gloves cannot justify unprotected patient contact. A separate room for the donning and doffing and storage of the necessary equipment is useful if available (Figure 1). Afterwards, the consultation room should be considered "contaminated" for 3 hours ${ }^{(7)}$. This should be indicated on the door, proper cleaning procedures and adequate personal protection of cleaning staff are required. Importantly, high level disinfection procedures should be followed for equipment like rigid and flexible endoscopes (List N: Disinfectants for Use Against SARS-CoV-2. United States Environmental Protection Agency. https://www.epa.gov/ 


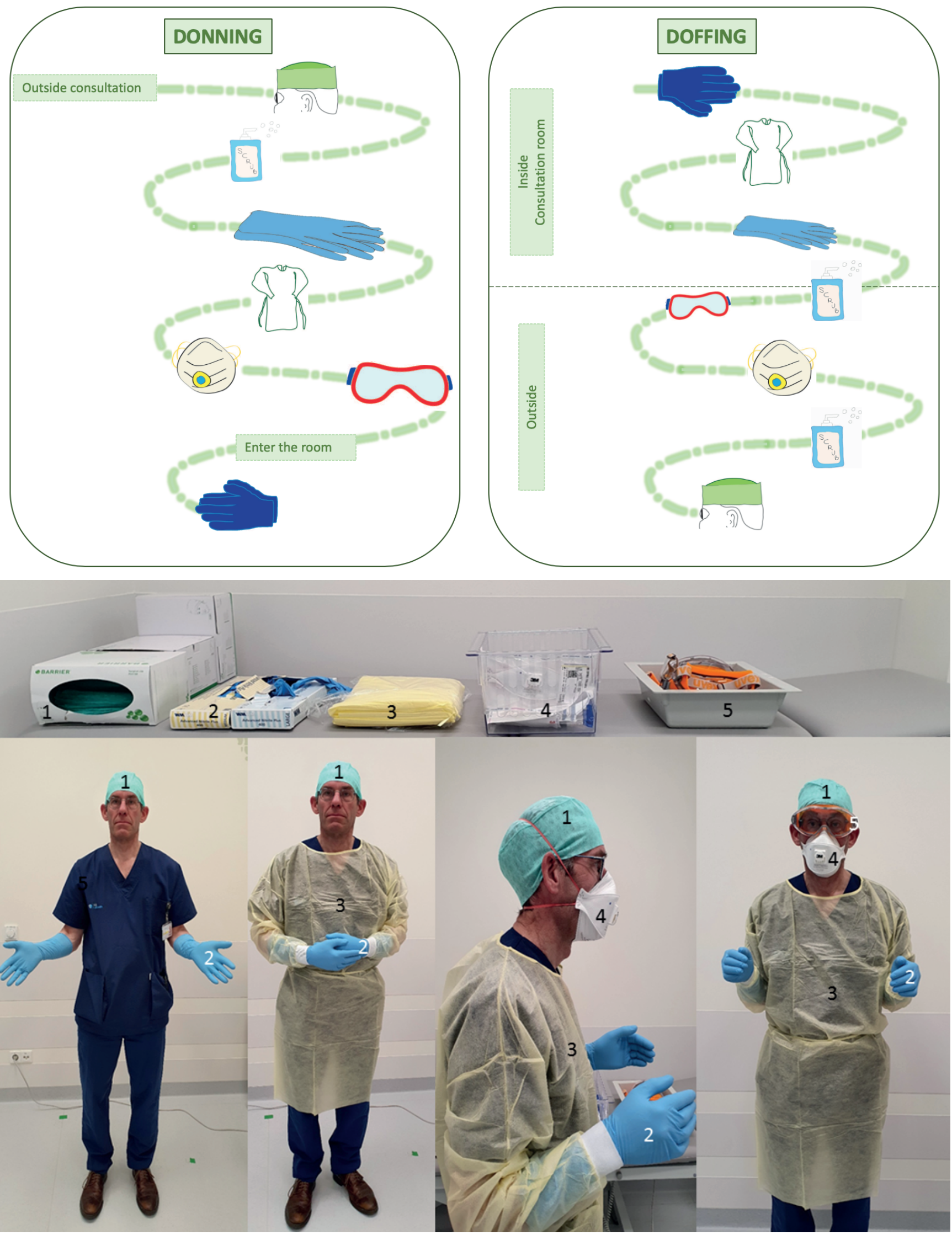

Figure 1. (A) Schematic figure of the donning and doffing procedure for personal protective equipment (PPE) in the outpatient rhinology clinic. (B) Illustration of the real-life PPE-procedure. 


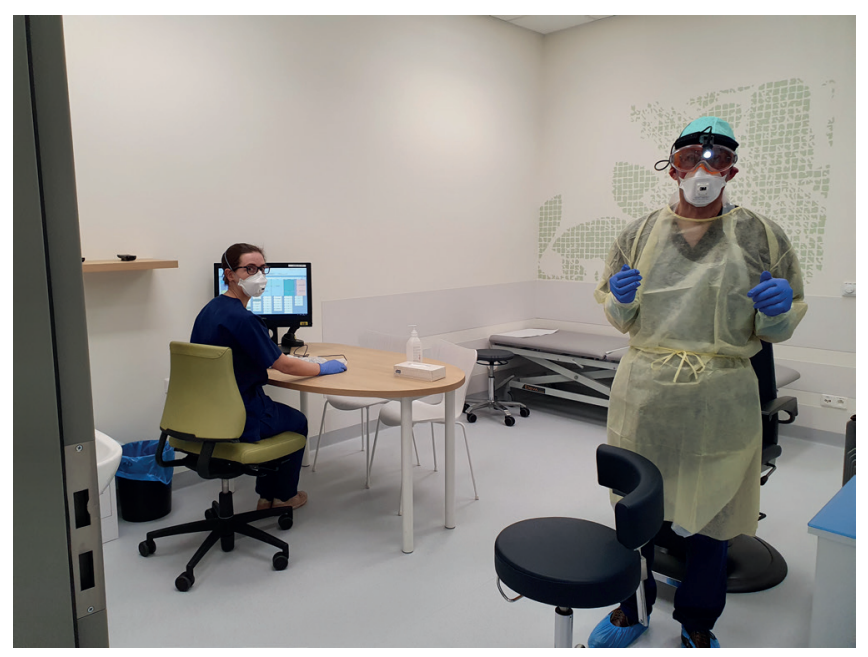

Figure 2. Real-life illustration of the examiner and buddy positioning with personal protective equipment.

pesticide-registration/list-n-disinfectants-useagainst-sars-cov-2. Published 2020).

To optimize the patient flow we suggest to organize the consultation in pairs with one physician being an experienced/ senior staff member who performs the clinical examination ("examiner") and an assistant who remains at distance from the patient ("buddy"). This person performs all other tasks, such as opening the medical file from the computer, writing down clinical findings, providing additional equipment if necessary and prescribing medication (Figure 2). It is important that the examiner remains within the area of examination and the buddy remains at a distance $(>1,5 \mathrm{~m})$. In this way exposure can be restricted in time and less PPE material is used since only upper (nitrile) gloves need to be changed between patients unless visible droplet spilling on the gown/goggles.

Since contamination is most likely to happen because of errors during the "undressing/doffing" procedure, leading to accidental contact with the contaminated mask, goggles/face visor or front of the gown, extra awareness and training for this procedure is advisable. Afterwards, it is important to put the used PPE material in the appropriate waste or recycle bin at the right location; gloves and gown inside the consultation room and goggles and mask outside.

\section{Summary outpatient rhinology clinic:}

- Provide patients with a mask.

- Question patients about COVID-19 contact and symptoms before clinical examination.

- Apply social distancing in the waiting area. Limit the waiting time and number of patients.

- Prepare material before starting the clinical examination.

- Limit the number of manipulations during clinical examination.

- Avoid the use of local anesthetic atomized sprays but use soaked pledgets instead.

- Use an endoscopy tower to avoid close physical contact.

- Allow only essential and experienced staff with proper PPE, work in pairs to optimize the patient flow and to reduce the need of PPE.

- Keep doors shut as much as possible.

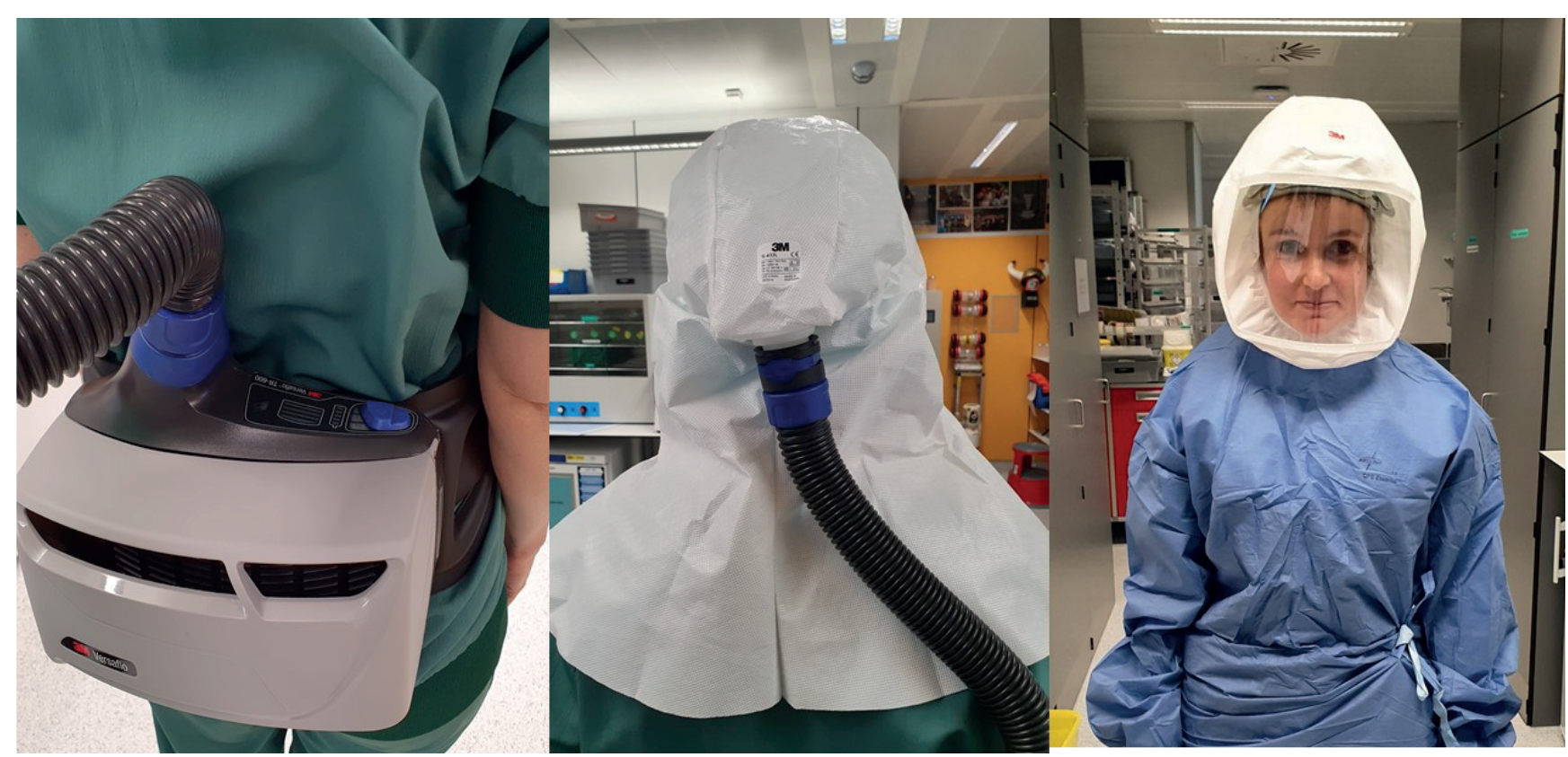

Figure 3. Real-life illustration of mask with powered, air-purifying respirator (PAPR), suggested as PPE for COVID-19 positive patients requiring endoscopic sinus surgery. 
Surgical endoscopic endonasal procedures including skull base surgery

All elective rhinologic procedures should be postponed until health care systems are starting to function normally again. Therefore, surgery should be limited to oncological cases and emergencies like intra-orbital and intracranial complications of acute rhinosinusitis, CSF-leaks, pituitary apoplexia or adenomas/ tumors with vision loss that cannot be postponed for months. For all semi-urgent elective cases, pre-operative screening of the COVID-19 status of the patient is recommended to adapt the PPE accordingly. In case of emergency where COVID-19 screening would imply an unacceptable time-delay, the patient should be considered as possibly COVID-19 positive and the same protective measures should be taken as for a confirmed COVID-19 positive patient ${ }^{(15)}$. Recurrent epistaxis with need to sphenopalatine artery ligation might be postponed until test results are known.

The screening method is highly variable in different centers due to resource allocation ${ }^{(16)}$. COVID-19 status can be determined based on clinical presentation in combination with real-time reverse-transcription polymerase chain reaction (RT-PCR) (nasopharyngeal swab) and/or chest multi-sliced computed tomography (MSCT) ${ }^{(17)}$. In case of doubt, for example negative PCR but symptoms and doubtful MSCT chest interpretation, maximal precautions are strongly recommended. We also advise to restrict the number of surgical team members and avoid the presence of trainees or observers in the operating room in order to save on PPE and increase the individual safety.

For endoscopic procedures the use of powered instruments like shaver and/or drill potentially increases the aerosol formation of the viral particles and should be avoided whenever possible. Considering transcranial surgery instead of endonasal surgery in particular cases of skull base pathology might be an alternative (safer) solution.

When there is an absolute need for endonasal endoscopic surgery in COVID-19 positive patients or patients with an unknown COVID-19 status, the operating room (OR) should be prepared and adapted accordingly; 1 ) the ventilation and pressure in the OR and corridor should be equalized (or OR in lower pressure). The usual "overpressure" mode can result in viral spreading from the OR towards the corridors with potential risk of infection of unprotected health care workers in the corridor, 2) OR entry doors should clearly indicate the COVID-19 positive status as well as the donning and doffing procedures to avoid accidental, unprotected entering of the $\mathrm{OR}, 3$ ) the anesthesiologist should wear proper PPE including an FFP3 mask and goggles to execute a save intubation ${ }^{(18,19)}$.
Recommendations for PPE for endoscopic endonasal procedures include for COVID-19 positive patients and emergencies, patients with unknown or doubtful COVID-19 status; classical surgical outfit (scrubs, OR shoes, cap), a mask with powered, air-purifying respirator (PAPR) and sterile gloves. It is important that the PAPR is switched on before entering the OR. For proven COVID-19 negative patients no special PPE is warranted (Summary see Table 1 ).

The use of this specific protective mask with powered, airpurifying respirator (PAPR, Figure 3), an enclosed powered system with HEPA filter for endoscopic sinus surgery and skull base surgery was recommended by Dr. Xiaoguang Tong, Professor and Chief of Neurosurgery at Tianjin Huanhu Hospital and leader of the Tianjin Emergency Medical Team deployed in Wuhan, China to combat the COVID-19 outbreak. They reported that the first case with the most widespread infection was an endoscopic pituitary surgery despite the use of FFP2 masks (documented via China Newsweek, View.inews. qq.com/a/20200125A07TT200?uid=\&devid=BDFE70CD-5BF1 4702-91B7-329F20A6E839\&qimei=bdfe70cd-5bf1-4702-91b7329f20a6e839).

\section{Summary endoscopic endonasal surgery:}

- Pre-operative screening of patients for COVID-19 status is strongly recommended.

- In case of unknown or positive COVID-19 status: o Consider alternative approaches to treat your patient. o Adapt ventilation and pressure in the OR. o Check procedure with anesthesiologist for COVID-19 intubation.

o Provide clear information on the OR doors and keep doors shut as much as possible.

o Limit the use of powered instruments.

o Allow only essential staff with proper PPE.

\section{Discussion}

The main goal of the authors was to provide a practical document for rhinologists' everyday practice, listing and summarizing the limited available data regarding PPE in this SARS-CoV-2 pandemic in a comprehensive way. The PPE recommendations are adapted to the nature of the procedure and the setting (outpatient clinic versus $\mathrm{OR}$ ) to maximize the usability of the document. In the near future, more widespread screening and the availability of antibody testing might change the current recommendations.

The question of which and whether two separate negative tests are needed before surgery, is still under active debate ${ }^{(16)}$.

The major limitation of these recommendations is the lack of strong supporting literature and research data. The short history 
of the COVID-19 outbreak and the onset in China with much (yet) untranslated literature are responsible for this shortcoming (20).

Moreover, guidelines regarding COVID-19 diagnosis and treatment are still under discussion and evolve almost daily with adjustments and implementations of new observations. Nevertheless, the authors strongly believe that any contribution to increase the awareness of the vulnerability of rhinologists is valuable at this time point of the crisis, which is most probably only the beginning of a prolonged period of adapted daily practice for rhinologists worldwide.

\section{Conclusion}

Transmission of SARS-CoV-2 is primarily through droplet spread, exposing rhinologists to a high reservoir of viral load as we are dealing with pathology in the nasal cavity, paranasal sinuses and rhinopharynx. Awareness and vigilance to protect ourselves and team members with the necessary PPE during our daily activity is of utmost importance to avoid spreading and accidental infection.

\section{Authorship contribution}

LVG is responsible for the concept of this paper, wrote the manuscript and made the figure and table outlines.

All other authors provided valuable feedback, suggestions and corrections to improve the quality of the manuscript.

\section{Conflict of interest}

The authors have no conflict of interest to declare regarding this paper.

\section{References}

1. Zhu N, Zhang D, Wang W et al. A Novel Coronavirus from Patients with Pneumonia in China, 2019. N. Engl. J. Med. 2020 20;382(8):727-33

2. Chen Y, Liu Q, Guo D. Emerging coronaviruses: Genome structure, replication, and pathogenesis. J. Med. Virol. 2020;92(4):41823

3. Wang C, Horby PW, Hayden FG, Gao GF. A novel coronavirus outbreak of global health concern. Lancet Lond. Engl. 2020 15;395(10223):470-3

4. Li Q, Guan X, Wu P et al. Early Transmission Dynamics in Wuhan, China, of Novel Coronavirus-Infected Pneumonia. N. Engl. J. Med. 2020 26;382(13):1199-207.

5. Zou L, Ruan F, Huang $M$ et al. SARS-CoV-2 Viral Load in Upper Respiratory Specimens of Infected Patients. N. Engl. J. Med. 2020 19:382(12):1177-9.

6. Tran K, Cimon K, Severn M, Pessoa-Silva $\mathrm{CL}$, Conly J. Aerosol generating procedures and risk of transmission of acute respiratory infections to healthcare workers: a systematic review. PloS One. 2012;7(4):e35797.

7. van Doremalen N, Bushmaker T, Morris $\mathrm{DH}$ et al. Aerosol and Surface Stability of SARS-CoV-2 as Compared with SARS-CoV-1. N Engl J Med. 2020 Mar 17. doi: 10.1056/ NEJMc2004973. [Epub ahead of print].

8. Vukkadala N, Qian ZJ, Holsinger FC, Patel ZM, Rosenthal E. COVID-19 and the otolaryngologist - preliminary evidence-based review. Laryngoscope. 2020 Mar 26. doi: 10.1002/lary.28672. [Epub ahead of print].

9. Workman A, Welling B, Carter, BS, et al Endonasal instrumentation and aerosolization risk in the era of COVID-19: simulation, literature review, and proposed mitigation strategies. Int Forum Allergy Rhinol. 2020 Apr 3. doi: 10.1002/alr.22577. [Epub ahead of print].

10. Lu D, Wang $H$, Yu R, Yang $H$, Zhao $Y$. Integrated infection control strategy to minimize nosocomial infection of coronavirus disease 2019 among ENT healthcare workers. JJ Hosp Infect. 2020 Feb 27. pii: S0195-6701(20)30092-X. doi: 10.1016/j. jhin.2020.02.018. [Epub ahead of print].

11. Gane SB, Kelly C, Hopkins C. Isolated sudden onset anosmia in COVID-19 infection. A novel syndrome? Rhinology. 2020 Apr 2. doi: 10.4193/Rhin20.114. [Epub ahead of print].

12. Brann DH, Tsukahara T, Weinreb C, Logan DW, Datta SR. Non-neural expression of SARS-CoV-2 entry genes in the olfactory epithelium suggests mechanisms underlying anosmia in COVID-19 patients. doi: https://doi.org/10.1101/2020.03.25.009084.

13. Gupta K, Mohanty SK, Kalra S, et al. The molecular basis of loss of smell in 2019nCoV infected individuals. https://doi. org/10.31219/osf.io/cmfsp

14. Kamming D, Gardam M, Chung $F$ Anaesthesia and SARS. Br. J. Anaesth. 2003 Jun;90(6):715-8.

15. Ti LK, Ang LS, Foong TW, Ng BSW. What we do when a COVID-19 patient needs an operation: operating room preparation and guidance. Can J Anaesth. 2020 Mar 6. doi: 10.1007/s12630-020-01617-4. [Epub ahead of print].

16. Ai T, Yang Z, Hou H et al. Correlation of Chest CT and RT-PCR Testing in Coronavirus Disease 2019 (COVID-19) in China: A Report of 1014 Cases. Radiology. 2020 Feb
26;200642.

17. Rodriguez-Morales AJ, Cardona-Ospina JA, Gutiérrez-Ocampo E et al. A systematic review and meta-analysis. Travel Med. Infect. Dis. 2020 Mar 13;101623.

18. Peng PWH, Ho P-L, Hota SS. Outbreak of a new coronavirus: what anaesthetists should know. Br J Anaesth. 2020 Feb 27. pii: S00070912(20)30098-2.

19. Zuo M-Z, Huang Y-G, Ma W-H et al. Recommendations for Tracheal Intubation in Critically ill Patients with Noval Coronavirus Disease 2019. Chin. Med. Sci. J. Chung-Kuo Hsueh Ko Hsueh Tsa Chih. 2020 Feb 27;

20. Xu K, Lai $X Q$, Liu Z. Suggestions for prevention of 2019 novel coronavirus infection in otolaryngology head and neck surgery medical staff. Zhonghua Er Bi Yan Hou Tou Jing Wai Ke Za Zhi. 2020 Feb 2;55(0):E001.

Prof. Dr. L. Van Gerven

Department of Otorhinolaryngology,

Head \& Neck Surgery

University Hospitals Leuven

Leuven

Belgium

Tel: +3216336390

E-mail: laura.vangerven@uzleuven.be 